TOKYo J. MATH.

VoL. 9, No. 2, 1986

\title{
Calabi Lifting and Surface Geometry in $S^{4}$
}

\author{
Norio EJIRI \\ Tokyo Metropolitan University
}

\section{Introduction}

Let $S^{4}(1)$ be the 4-dimensional unit sphere. Fix an orientation of $S^{4}(1)$. We denote by $T_{x}$ the space of orthogonal complex structures compatible with the orientation of $T_{x}\left(S^{4}(1)\right)$ and by $T=\cup_{x \in S^{4}(1)} T_{x}$ the fiber bundle over $S^{4}(1) . \quad T$ is called the twistor space of $S^{4}(1)$ and it is well-known that $T$ is the 3 -dimensional complex projective space $P_{3}$ and the projection of $T$ onto $S^{4}(1)$ is the Hopf fibration. Let $M$ be an orientable Riemannian surface isometrically immersed in $S^{4}(1)$ and $J^{1}$ a complex structure compatible with a fixed orientation of $M$. Then the normal space $N_{x}(M)$ admits an orthogonal complex structure $J_{x}^{2}$ defined by $J_{x}^{1}$ and the orientation of $S^{4}(1)$ and hence $J_{x}^{1}+J_{x}^{2}$ is an orthogonal complex structure of $T_{x}\left(S^{4}(1)\right)$ for $x \in M$, where $J_{x}^{1}$ is the orthogonal complex structure of $T_{x}(M)$ given by $J^{1}$. Therefore we obtain a map of $M$ into $P_{3}$ defined by $x \in M \rightarrow J_{x}^{1}+J_{x}^{2} \in P_{3}$. We call this map the Calabi lifting defined by the fixed orientation of $S^{4}(1)$ ([19]). Choosing the reverse orientation of $S^{4}(1)$, we have another Calabi lifting. We call the former the positive Calabi lifting and the latter the negative Calabi lifting. If $M$ is an orientable surface in the 4-dimensional Euclidean space $R^{4}$, we can define the Gauss map of $M$ into $S^{2} \times S^{2}$. We note that the Gauss map is constructed as follows: By the same argument as above, each point of $M$ gives an orthogonal complex structure of $R^{4}$ compatible with the fixed orientation of $R^{4}$. Since the space of orthogonal complex structures of $R^{4}$ is $S^{2}$, we obtain a map of $M$ into $S^{2}$. Changing the orientation of $R^{4}$, we get another map of $M$ into $S^{2}$. It is easy to see that these maps of $M$ into $S^{2}$ give the Gauss map of $M$ into $S^{2} \times S^{2}$. From this point of view, we may regard Calabi lifting as "Gauss map".

In this paper, we investigate some relations between an isometric immersion into $S^{4}(1)$ and its Calabi lifting. In the sections 1,2 and 3, we review the results of Chern [7] and Barbosa [2] on minimal surfaces 
of $S^{4}(1)$, the results of Lawson [13] on the Frenet frame field of a holomorphic curve in $P_{8}$ and the results of Eells and Wood [9] on the isotropic harmonic maps into $P_{3}$. In the sections $4, \cdots, 7$, we give the summary of the definition and properties of the twistor space of $S^{4}(1)$. In the section 8 , we define the Calabi lifting of an orientable Riemannian surface $M$ isometrically immersed in $S^{4}(1)$. Let $d_{1}$ and $d_{2}$ be the degrees of the positive and negative Calabi liftings. We denote by $\chi(M)$ and $\chi(N(M))$ the Euler numbers of $M$ and its normal bundle $N(M)$, respectively. Then we obtain

$$
d_{1}+d_{2}=\chi(M) \text { and } d_{1}-d_{2}=\chi(N(M)) .
$$

This formula is well-known on surfaces in $R^{4}$ (see, for example, [8]). Furthermore the energy $E$ of the Calabi lifting is given by

$$
E=4 \int\left(1+|\mathfrak{h}|^{2}\right)^{*} 1-4 \pi(\chi(M)+\chi(N(M))),
$$

where $\mathfrak{h}$ is the mean curvature vector of $M$. In the section 9 , we can prove that the positive Calabi lifting is holomorphic if and only if the ellipse of curvature (See, for example, [11].) is a positive circle. This fact is obtained by Atiyah and Lawson (See [11].) and, together with the formula of $E$, gives a result in [11] in the case that the ambient space is $S^{4}(1)$. Furthermore, we see that the positive Calabi lifting of a superminimal surface is holomorphic and horizontal. In the section 10 , we can prove that the constancy of "the second curvature" of a holomorphic curve in $P_{3}$ is equivalent to its horizontality. In the section 11 , we can prove that the positive Calabi lifting is harmonic if and only if the mean curvature vector of $M$ is a holomorphic cross section of the normal bundle. In particular, each Calabi lifting of an orientable minimal surface in $S^{4}(1)$ is a conformal minimal immersion into $P_{3}$. In the Section 12 , we can show that the positive Calabi lifting is a non-holomorphic isotropic harmonic map if and only if the negative Calabi lifting is holomorphic and horizontal and hence $M$ is a superminimal surface of $S^{4}(1)$. Furthermore the nonholomorphic isotropic harmonic map is constructed from the negative Calabi lifting by the method of Eells and Wood [9] etc. Using the result of [9], we see that, for a minimal surface $T^{2}$ of genus 1, if the Euler number of $N\left(T^{2}\right)$ is not zero, $T^{2}$ is a superminimal surface. We generalize this fact as follows: Let $M$ be a compact orientable minimal surface of genus $p$ of $|\chi(N(M))| \geqq 2 p-1$. Then $M$ is a superminimal surface.

Let $M$ be a holomorphic curve in $P_{8}$ such that the image of $M$ by the Hopf fibration is a regular surface $N$. Then 


$$
\int\left(1+|\mathfrak{h}|^{2}\right)^{*} 1
$$

attains the minimum among the regularly homotopic immersions of $N$ into $S^{4}(1)$ [11]. However, in general, the centroid of $M$ is not zero. On the other hand, Weiner posed a question wheather the only closed orientable surfaces immersed in $S^{n}(1)$ with centroid 0 which satisfy the Euler-Lagrange equation of $(0.1)$ are minimal surfaces in $S^{n}(1)$. In spite of a counter example given in [10], it is interesting to consider this question in a restricted case. But we see that this question is negative even in the restricted case.

In the section 15, we give an application of a holomorphic, horizontal curve in $P_{3}$. From [12], we note that a cone of an $n$-dimensional submanifold $M$ in $S^{2 n+1}(1)$ is special Lagrangian ([12]) if and only if $M$ is an integral, minimal submanifold ([3]) in $S^{2 n+1}(1)$. On the other hand, it is well-known that the image of an $n$-dimensional integral, minimal submanifold $M$ by Hopf fibration $S^{2 n+1}(1) \rightarrow P_{n}(4)$ is totally real and minimal ([6]) in $P_{n}(4)$. Conversely, for a simply connected $n$-dimensional totally real, minimal submanifold $M$ in $P_{n}(4)$, there exists a lifting map of $M$ into $S^{2 n+1}(1)$ which gives an integral, minimal submanifold such that its image by Hopf fibration is a totally real, minimal submanifold. Thus it is very interesting to study $n$-dimensional totally real, minimal submanifolds in $P_{n}$. We can prove that the circle bundle of $S^{2}$ of positive even Chern number $(\geqq 6)$ can be immersed in $P_{3}$ as a totally real, minimal submanifold.

The author expresses his deep gratitude to Professor K. Ogiue who encouraged him and gave him a lot of valuable suggestions.

\section{§1. Elementary surface geometry.}

Let $M$ be a Riemannian surface with a fixed orientation isometrically immersed in $S^{4}(1)$ and $\chi$ the immersion. Let $J^{1}$ be a complex structure compatible with the fixed orientation of $M$. We denote by $\nabla, \bar{\nabla}$ and $\nabla^{N}$ the connections of $T(M), T\left(S^{4}(1)\right)$ and the normal bundle $N(M)$ of $M$ in $S^{4}(1)$, respectively. Let $\sigma$ be the second fundamental form of $M$ given by

$$
\sigma(X, Y)=\bar{\nabla}_{X} Y-\nabla_{X} Y
$$

and $\nabla^{\prime} \sigma$ the differentiation of $\sigma$ defined by

$$
\left(\nabla_{X}^{\prime} \sigma\right)(Y, Z)=\nabla_{X}^{N} \sigma(Y, Z)-\sigma\left(\nabla_{X} Y, Z\right)-\sigma\left(Y, \nabla_{X} Z\right) .
$$

Then the Gauss, Ricci and Codazzi equations are given by 


$$
\begin{gathered}
K\{\langle X, Z\rangle\langle Y, W\rangle-\langle X, W\rangle\langle Y, Z\rangle\}=\langle X, Z\rangle\langle Y, W\rangle-\langle X, W\rangle\langle Y, Z\rangle \\
+\langle\sigma(X, Z), \sigma(Y, W)\rangle-\langle\sigma(X, W), \sigma(Y, Z)\rangle, \\
\left\langle R_{X Y \xi}^{N}, \eta\right\rangle=\sum_{j=1}^{2}\left\{\left\langle\xi, \sigma\left(X, e_{j}\right)\right\rangle\left\langle\eta, \sigma\left(Y, e_{j}\right)\right\rangle\right. \\
\left.-\left\langle\xi, \sigma\left(Y, e_{i}\right)\right\rangle\left\langle\eta, \sigma\left(X, e_{i}\right)\right\rangle\right\}, \\
\left(\nabla_{X}^{\prime} \sigma\right)(Y, Z)=\left(\nabla_{Y}^{\prime} \sigma\right)(X, Z),
\end{gathered}
$$

where $K$ is the Gauss curvature of $M, R^{N}$ is the curvature tensor of $\nabla^{N}$ defined by $R_{X Y}^{N}=\nabla_{[X, Y]}^{N}-\nabla_{X}^{N} \nabla_{Y}^{N}+\nabla_{Y}^{N} \nabla_{X}^{N}$ and $\left\{e_{1}, e_{2}\right\}$ is an oriented orthonormal basis of $M$. We denote by $K_{N}$ the normal curvature with respect to the given orientation of $N(M)$.

Now we fix the orientation of $S^{4}(1)$. Let $e_{1}, e_{2}, e_{3}, e_{4}$ be an oriented orthonormal basis of $S^{4}(1)$ and $e_{1}, e_{3}$ the oriented basis of $M$. Then $N(M)$ has the natural orientation determined by the orientations of $M$ and $S^{4}(1)$ and we can define an orthogonal complex structure $J^{2}$ of $N(M)$ by

$$
J^{2} e_{2}=e_{4} \text { and } J^{2} e_{4}=-e_{2} \text {. }
$$

It is easy to see that

$$
\nabla^{\prime} J^{2}=0,
$$

which gives the Hermitian connection of $N(\boldsymbol{M})$ such that $N(\boldsymbol{M})$ is a holomorphic line bundle (see, for example, [9, page 221]). Let $\xi$ be a cross section of $N(M)$ and $D$ the operator of $N(M)$ defined by

$$
D_{x \xi}=J^{2} \nabla_{X}^{N} \xi-\nabla_{J^{1} X}^{N} \xi .
$$

Then $\xi$ is holomorphic if and only if $D \xi=0$. We put $h_{j k}^{\alpha}=\left\langle\sigma\left(e_{j}, e_{k}\right), e_{\alpha}\right\rangle$ for $j, k=1,3$ and $\alpha, \beta=2,4$. For a point $x$ of $M$, we define a map of the unit circle $S_{x}^{1}$ of $T_{x}(M)$ into $R_{+}$by $X \rightarrow\|\sigma(X, X)\|^{2}$. Let $e_{1}$ be a unit vector at which the function attains a maximum and $e_{3}=J^{1} e_{1}$. Then if $\sigma\left(e_{1}, e_{1}\right)$ is non-zero, we can take the unit normal vector $e_{2}$ as

$$
e_{2}=\frac{\sigma\left(e_{1}, e_{1}\right)}{\left\|\sigma\left(e_{1}, e_{1}\right)\right\|}
$$

and $e_{4}=J^{2} e_{2}$. With respect to the basis, we obtain

$$
h_{j k}^{2}=\left|\begin{array}{cc}
\lambda & 0 \\
0 & \mu
\end{array}\right|, \quad h_{j k}^{4}=\left|\begin{array}{ll}
0 & \nu \\
\nu & \delta
\end{array}\right|
$$

and $\lambda \neq 0$. If $\sigma\left(e_{1}, e_{1}\right)=0$, then $x$ is a geodesic point and hence we may 
consider $\lambda=\mu=\nu=\delta=0$. We call this basis an $E$-frame. We easily see that $K=1+\lambda \mu-\nu^{2}$ and $K_{N}=\nu(\lambda-\mu)$. Next we define the map of $S_{x}^{1}$ into $N_{x}(M)$ by $X \rightarrow \sigma(X, X)$ whose image is called the ellipse of curvature (see, for example, [11] and [20]). We call the map the ellipse of curvature in this paper.

LEMMA 1.1. The image of the ellipse of curvature is a circle if and only if

$$
\nu= \pm \frac{\lambda-\mu}{2} .
$$

In particular, the ellipse of curvature preserves or reverses the orientation according as $\nu=(\lambda-\mu) / 2$ or $\nu=-(\lambda-\mu) / 2$.

The ellipse of curvature preserving (resp. reversing) the orientation is called the positive (resp. negative) circle. In particular a minimal surface in $S^{4}(1)$ is called superminimal [4] or $R$-surface [20] if and only if the image of the ellipse of curvature is a positive circle.

\section{§2. Superminimal surfaces in $S^{4}(1)$.}

We review the results of Chern [7] and Barbosa [2] on a superminimal surface in $S^{4}(1)$.

Let $M$ be a superminimal surface in $S^{4}(1)$ and $\chi$ the immersion. Let $z$ be an isothermal coordinate of $M$ and (, ) the symmetrical product in the 5-dimensional complex Euclidean space $C^{5}$. Then vector valued functions on $M$ are locally defined by

$$
\begin{aligned}
& G_{0}=\chi \\
& G_{1}=\bar{\partial} \chi \\
& G_{2}=\bar{\partial}^{2} \chi-a G_{1},
\end{aligned}
$$

where $a$ is choosen in such a way that $\left(G_{2}, \bar{G}_{1}\right)=0$. They satisfy the following.

Lemma 2.1 ([2, (3.7) Lemma, page 80]).

$$
\begin{gathered}
\bar{\partial} G_{1}=G_{2}+\left(\bar{\partial} \log \left|G_{1}\right|^{2}\right) G_{1}, \\
\partial G_{1}=-\left|G_{1}\right|^{2} G_{0}, \\
\bar{\partial} G_{2}=\left(\bar{\partial} \log \left|G_{2}\right|^{2}\right) G_{2}
\end{gathered}
$$

and hence $\xi=G_{2} /\left|G_{2}\right|^{2}$ is holomorphic. In particular, $\xi$ satisfies 


$$
(\xi, \xi)=\left(\xi^{\prime}, \xi^{\prime}\right)=0
$$

LEMMA 2.2 ([2, (3.12) Proposition, page 81]). $\xi$ has at most isolated singularities with poles and gives a holomorphic map $\Xi$ of $M$ into $P_{4}$ which is called the directrix curve.

We define $\psi$ by $\xi \wedge \xi^{\prime} \wedge \bar{\xi} \wedge \bar{\xi}^{\prime}$. Since $\wedge^{4} C^{5}$ is $C^{5}, \psi$ is parallel to $\chi$. Conversely let $\xi$ be a full holomorphic map of $M$ into $P_{4}$ whose local expression satisfies (2.1) (it is called totally isotropic). Then we can construct $\psi$ and obtain the following.

Proposition 2.1 ([2, (3.7) and (3.8) Propositions, pages 83, 85]). $\psi /|\psi|$ is independent of $z$ and gives the global map $\chi$ of $M$ into $S^{4}(1)$ and

$$
(\partial \chi, \bar{\partial} \chi)=\frac{\left|\xi_{1} \wedge \xi_{1}^{\prime}\right|^{2}}{\left|\xi_{1}\right|^{4}},
$$

where $\xi_{1}=\xi \wedge \xi^{\prime}$ (it gives holomorphic map of $M$ into $P_{9}$ which is called the first associated holomorphic curve of $\Xi$ ).

Generally $\chi$ constructed as above is a branched immersion (see, for example, [2]). Consequently we obtain

TheOREM 2.1 ([2, (3.30) Theorem, page 88]). There exists a canonical 1-1 correspondence between the set of full superminimal branched immersions $\chi$ of $M$ into $S^{4}(1)$ and the set of full and totally isotropic holomorphic maps $\xi$ of $M$ into $P_{4}$. The correspondence is the one that associates with a superminimal immersion $\chi$ its directrix curve.

Since $M$ is superminimal, we obtain $\lambda=\nu, \mu=-\lambda$ and $\sigma=0$ for $E$ frame $e_{i} i=1, \cdots, 4$. Furthermore we obtain

LEMMA 2.3.

$$
G_{1}=\frac{\rho}{2} \bar{E}_{1} \quad \text { and } \quad G_{2}=\frac{\rho^{2}}{2} \lambda \bar{E}_{2},
$$

where $\rho^{2} d z d \bar{z}$ is the metric and $E_{1}=e_{1}-i e_{3}, E_{2}=e_{2}-i e_{4}$.

\section{§3. Holomorphic curves in $P_{3}$ and isotropic maps into $P_{3}$.}

Let $M$ be a compact orientable Riemannian surface fully and holomorphically immersed in $P_{3}$. Then, except for isolated points of $M$, we can define the second and third fundamental forms $\sigma_{2}$ and $\sigma_{3}$, respectively. We easily obtain $l_{1}$ and $l_{2}$ such that

$$
\left\langle\sigma_{2}\left(e_{1}, e_{1}\right), \sigma_{2}\left(e_{1}, e_{2}\right)\right\rangle=0 \text {, }
$$




$$
\begin{gathered}
\left\|\sigma_{2}\left(e_{1}, e_{1}\right)\right\|=\left\|\sigma_{2}\left(e_{2}, e_{2}\right)\right\|=l_{1} \neq 0, \\
\left\langle\sigma_{3}\left(e_{1}, e_{1}, e_{1}\right), \sigma_{3}\left(e_{1}, e_{1}, e_{2}\right)\right\rangle=0, \\
\left\|\sigma_{3}\left(e_{1}, e_{1}, e_{1}\right)\right\|=\left\|\sigma_{3}\left(e_{1}, e_{1}, e_{2}\right)\right\|=l_{2} \neq 0,
\end{gathered}
$$

where $\left\{e_{1}, e_{2}\right\}$ is an oriented orthonormal frame field of $M$. Set

$$
\begin{gathered}
e_{3}=\sigma_{2}\left(e_{1}, e_{1}\right) / l_{1}, \quad e_{4}=\sigma_{2}\left(e_{1}, e_{2}\right) / l_{1}=J \sigma_{2}\left(e_{1}, e_{1}\right) / l_{1}, \\
e_{5}=\sigma_{3}\left(e_{1}, e_{1}, e_{1}\right) / l_{2},
\end{gathered}
$$

and $\kappa_{1}=l_{1}$ and $\kappa_{2}=l_{2} / l_{1}$. Then we have

$$
\begin{aligned}
& \tilde{\nabla} e_{2 s-1}=-\kappa_{s-1} \omega_{1} e_{2 s-3}+\kappa_{s-1} \omega_{2} e_{2 s-2}+\omega_{2 s-1,2 s} e_{2 s}+\kappa_{s} \omega_{1} e_{2 s+1}+\kappa_{s} \omega_{2} e_{s+2} . \\
& \tilde{\nabla} e_{2 s}=-\kappa_{s-1} \omega_{1} e_{2 s-2}-\kappa_{s-1} \omega_{2} e_{2 s-3}-\omega_{2 s-1,2 s} e_{2 s-1}+\kappa_{s} \omega_{1} e_{2 s+2}-\kappa_{s} \omega_{2} e_{2 s+1},
\end{aligned}
$$

where $s=1,2, \kappa_{0}=\kappa_{s}=0$ and $\omega_{2 s-1,2 s}\left(e_{j}\right)=\left\langle\tilde{V}_{e j} e_{2 s-1}, e_{2 s}\right\rangle$. We call $\kappa_{s}$ the $s$-th curvature of $M$. Next we put $\theta_{s-1}=\omega_{2 s-1,2 s}-s \omega_{1,2}$. Then

$$
\theta_{s}=d^{\circ} \log \kappa_{1} \cdots \kappa_{s}
$$

holds and we get

$$
\begin{gathered}
\frac{1}{2} \Delta \log \left(\kappa_{1} \cdots \kappa_{s}\right)+\kappa_{s}^{2}-\kappa_{s+1}^{2}-\frac{s+1}{2} K+\frac{1}{4}=0 \text { for } s=1,2, \\
\kappa_{1}^{2}=\frac{1}{2}(1-K) .
\end{gathered}
$$

For these results, see [13, page 92]. We remark that $\kappa_{\varepsilon}^{2}$ is equel to $K_{s}$ given in [13].

LEMMA 3.1. If $\kappa_{2}$ is constant, then $\kappa_{2}=1 / 2$.

Next we review the results of Eells and Wood [9].

Let $f$ be a full holomorphic map of $M$ into $P_{3}$ and $\left(z_{0}, z_{1}, z_{2}, z_{3}\right)$ the homogeneous coordinates of $P_{3}$ and $z$ an isothermal coordinate on an open set $U$ of $M$. We define $f_{U}: U \rightarrow C^{4}$ such that $f(x)=\left\{f_{U}(x)\right\} \in P_{3}\left(f_{U}\right.$ is called a local lift of $f$ ). Let $f_{1}$ and $f_{2}$ be the associated curves of $f$ defined by $\left\{f_{U}, \partial f_{U}\right\}$ and $\left\{f_{U}, \partial f_{U}, \partial^{2} f_{U}\right\}$, respectively, and $g$ the polar of $f$ [9, page 226] defined by $f_{2}$. Then $\phi(x)=f_{1}(x)^{\perp} \cap f_{2}(x)$ or $f(x)^{\perp} \cap f_{1}(x)$ gives a full harmonic map of $M$ into $P_{3}$, where $f(x)^{\perp}$ is the subspace orthogonal to $f(x)$, etc. On the other hand, let $\varphi$ be a map of $M$ into $P_{3}$. Then $\varphi$ is called the isotropic map if 


$$
\left\langle D^{\prime \alpha} \varphi, D^{\prime \prime \beta} \varphi\right\rangle=0 \text { for } \alpha \text { and } \beta \geqq 1 \text {, }
$$

where $\langle$,$\rangle is the metric and D$ is the connection of $\varphi^{-1} T^{\prime} P_{3}\left(T^{\prime} P_{3}\right.$ is the holomorphic tangent bundle of $P_{3}$ ) and $D^{\prime}=D_{\partial / \partial z}, D^{\prime \prime}=D_{\partial / \partial \bar{\varepsilon}}$.

Proposition 3.1 ([9, Lemma 3.7, page 226]). $\phi$ is isotropic.

Conversely let $\phi$ be a full isotropic harmonic map of $M$ into $\mathrm{P}_{3}$. Then $D^{\prime}$-order $s$ and $D^{\prime \prime}$-order $r$ are defined by

$$
\begin{aligned}
& s=\operatorname{Max}_{x \in M} \operatorname{dim} \operatorname{span}\left\{D^{\prime \alpha} \phi: 1 \leqq \alpha<\infty\right\}, \\
& r=\operatorname{Max}_{x \in M} \operatorname{dim} \operatorname{span}\left\{D^{\prime \prime \alpha} \phi: 1 \leqq \alpha<\infty\right\},
\end{aligned}
$$

respectively.

Proposition 3.2 ([9, Lemma 6.2, page 240]).

$$
r+s=3 \text {. }
$$

It is easy to define the maps $\phi_{s}^{\prime}$ and $\phi_{r}$ of $M$ into the $s$ and $r$-dimensional complex Grassmann manifolds $G_{s}\left(C^{4}\right)$ and $G_{r}\left(C^{4}\right)$ by

$$
\begin{aligned}
& \phi_{s}^{\prime}(x)=\left\{D^{\prime} \phi, D^{\prime 2} \phi, \cdots, D^{\prime s} \phi\right\} \quad \text { and } \\
& \phi_{r}^{\prime \prime}(x)=\left\{D^{\prime \prime} \phi, D^{\prime \prime 2} \phi, \cdots, D^{\prime \prime r} \phi\right\},
\end{aligned}
$$

respectively. Furthermore maps $\tilde{\phi}_{s}^{\prime}$ and $\tilde{\phi}_{r}$ of $M$ into $G_{s+1}\left(C^{4}\right)$ and $G_{r+1}\left(C^{4}\right)$ are defined by $\phi \oplus \phi_{s}^{\prime}$ and $\phi \oplus \phi_{r}^{\prime \prime}$, respectively. Then we have the following.

TheOREM 3.1 ([9, Theorem 6.9, page 244)]. Let $M$ be a Riemannian surface. Then the set of full isotropic harmonic maps $\phi$ of $M$ into $P_{8}$ and the set of pairs of full holomorphic map $f$ and integer $r(0 \leqq r<3)$ have the bijective correspondence as follows:

$$
\begin{gathered}
r=D^{\prime \prime} \text {-order of } \phi \text { and } f=\tilde{\phi}_{r-1}^{\prime \prime} \cap \tilde{\phi}_{r}^{\prime \prime} \\
\phi=f_{r-1}^{\perp} \cap f_{r} .
\end{gathered}
$$

§4. Orthogonal complex structures of $R^{4}$.

Let $R^{4}$ be the 4-dimensional Euclidean space with a fixed orientation and $\left(x^{1}, x^{2}, x^{8}, x^{4}\right)$ oriented orthogonal coordinates of $R^{4}$. Let $J_{0}$ be an orthogonal complex structure defined by

$$
J_{0}=\left(\begin{array}{rr}
0 & -I \\
I & 0
\end{array}\right),
$$


where $I$ is the $2 \times 2$-identity matrix. Then the orientation is compatible with $J_{0}$. Let $S O(4)$ be the special orthogonal group and 0 the set of orthogonal complex structures compatible with the fixed orientation of $R^{4}$. Then $S O(4)$ acts transitively on 0 as the adjoint action

$$
J \longrightarrow g J g^{-1} \text {. }
$$

Since the isotropy group is the unitary group $U(2)$, we obtain $0=$ $S O(4) / U(2)$. We denote by $s o(4)$ and $u(2)$ the Lie algebras of $S O(4)$ and $U(2)$, respectively, which are given by

$$
\begin{aligned}
& \text { so }(4)=\left\{\left(\begin{array}{ll}
A & C \\
-{ }^{t} C & B
\end{array}\right), \quad \text { where } A \text { and } B \text { are skew-symmetric } 2 \times 2\right. \text {-matrices } \\
& C \text { is a } 2 \times 2 \text {-matrix }\} \text {, } \\
& u(2)=\left\{\left(\begin{array}{rr}
A & -B \\
B & A
\end{array}\right), \quad \text { where } A \text { is a skew-symmetric } 2 \times 2\right. \text {-matrix and } \\
& B \text { is a symmetric } 2 \times 2 \text {-matrix }\} \text {. }
\end{aligned}
$$

We have the canonical decomposition

$$
s o(4)=u(2)+\mathfrak{p},
$$

where $\mathfrak{p}$ if given by

$$
\mathfrak{p}=\left\{\left(\begin{array}{rr}
A & B \\
B & -A
\end{array}\right), \quad \text { where } A \text { and } B \text { are skew-symmetric } 2 \times 2 \text {-matrices }\right\} .
$$

Since the metric $\|\quad\|$ and the complex structure $J$ on $\mathfrak{p}$ are given by

$$
\begin{gathered}
\left\|\left(\begin{array}{rr}
A & B \\
B & -A
\end{array}\right)\right\|^{2}=-2 \operatorname{tr}\left(A^{2}+B^{2}\right), \\
J\left(\begin{array}{rr}
A & B \\
B & -A
\end{array}\right)=\left(\begin{array}{rr}
-B & A \\
A & B
\end{array}\right),
\end{gathered}
$$

we obtain the $S O(4)$-equivariant metric and complex structure of $S O(4) / U(2)$ and it is easy to see that $S O(4) / U(2)$ is $S^{2}(1)$. The reverse orientation of $R^{4}$ gives another equivalence between $S O(4) / U(2)$ and $S^{2}(1)$. These equivalences are given by 


$$
\begin{aligned}
& S O(4) / U(2) \longrightarrow S^{2}(1) \text {, } \\
& \left(e_{1}, e_{2}, e_{3}, e_{4}\right) U(2) \longrightarrow\left(\begin{array}{c}
\left\langle e_{1} \wedge e_{3}, E_{1} \wedge E_{2} \pm E_{8} \wedge E_{4}\right\rangle \\
\left\langle e_{1} \wedge e_{3}, E_{1} \wedge E_{8} \mp E_{2} \wedge E_{4}\right\rangle \\
\left\langle e_{1} \wedge e_{8}, E_{1} \wedge E_{4} \pm E_{2} \wedge E_{8}\right\rangle
\end{array}\right)
\end{aligned}
$$

where $E_{j}=(\ldots, 0,1,0, \ldots)$.

§5. Twistor space $P_{3}$ of $S^{4}(1)$.

Fix an orientation of $S^{4}(1)$. Let $F$ be the oriented frame bundle of $S^{4}(1)$ compatible with the orientation. Then, using the $S O(4)$-action on 0 , we construct the associated bundle $H$. We denote by $\left(x, e_{1}, e_{2}, e_{3}, e_{4}\right) U(2)$ an element of $H$. We obtain the diagram:

$$
\left.\left(x, e_{1}, \cdots, e_{4}\right) U(2) \in H \sum_{S^{4}(1) \ni x .}^{\pi /}\right|_{j \ni\left(x, e_{1}, \cdots, e_{4}\right)} ^{\pi}
$$

For $x \in S^{4}(1)$, the fiber $H_{x}$ of $H$ gives the space of orthogonal complex structures of $T_{x}\left(S^{4}(1)\right)$. Let $\mathscr{C}$ be the horizontal distribution of $H$ defined by the connection of $F$. Then, for $p \in H$, identifying $T_{\pi(p)} S^{4}(1)$ with $\mathscr{C}_{p}$, we obtain the innerproduct and the orthogonal complex structure of $\mathscr{K}_{p}$. Since the fiber has the metric and the orthogonal complex structure induced by 0 , we can define the almost Hermitian structure of $H$. The following fact is well-known ([1]).

$H$ is the 3-dimensional complex projective space $P_{3}$ of holomorphic sectional curvature 1 and $\pi$ is the Hopf fibration.

We note that $F=S O(5)$ and $H$ is the reductive homogeneous space $S O(5) / 1 \times U(2)$. In fact, let $s o(5)$ be the Lie algebra of $S O(5)$. Then the Lie algebra $1 \times u(2)$ of $1 \times U(2)$ given by

$$
\left\{\left(\begin{array}{rrr}
0 & & \\
& A & B \\
-B & A
\end{array}\right): \quad \begin{array}{l}
A \text { is a skew-symmetric } 2 \times 2 \text {-matrix } \\
\text { and } B \text { is a symmetric } 2 \times 2 \text {-matrix }
\end{array}\right\} .
$$

Let $\tilde{\mathfrak{p}}$ be

$$
\left.\left\{\begin{array}{rrr}
0 & \xi & \eta \\
-{ }^{t} \xi & A & C \\
-{ }^{t} \eta & C & -A
\end{array}\right): A \text { and } C \text { are skew-symmetric } 2 \times 2 \text {-matrices }\right\} .
$$


Then $s o(5)=1 \times u(2)+\tilde{p}$ and $S O(5) / 1 \times U(2)$ is a reductive homogeneous space. The metric and the complex structure are given by

$$
\begin{gathered}
\left\|\left(\begin{array}{rrr}
0 & \xi & \eta \\
-{ }^{t} \xi & A & C \\
-{ }^{t} \eta & C & -A
\end{array}\right)\right\|^{2}=|\xi|^{2}+|\eta|^{2}+2|A|^{2}+2|C|^{2} \text { and } \\
J\left(\begin{array}{rrr}
0 & \xi & \eta \\
-{ }^{t} \xi & A & C \\
-{ }^{t} \eta & C & -A
\end{array}\right)=\left(\begin{array}{rrr}
0 & -\eta & \xi \\
{ }^{t} \eta & -C & A \\
-{ }^{t} \xi & A & C
\end{array}\right) .
\end{gathered}
$$

$P_{3} \rightarrow S^{4}(1)$ is the Riemannian submersion (See, for example, [16].) and the fundamental tensor $A$ is given by

$$
\left\langle A_{\tilde{X}} \tilde{Y}, V\right\rangle=-\frac{1}{2}\left\langle\left(\left\langle R_{X Y} e_{j}, e_{k}\right\rangle\right)^{p}, V\right\rangle,
$$

where $\tilde{X}$ and $\tilde{Y} \in \mathscr{H}_{p}$ are the horizontal lifts of $X$ and $Y \in T_{\pi(p)} S^{4}(1), V$ is an element of the tangent space of the fiber (vertical vector) at $p$ identified with $\mathfrak{p}, p=\left(\pi(p), e_{1}, \cdots, e_{4}\right) U(2) ;\left(\left\langle R_{X Y} e_{j}, e_{k}\right\rangle\right)$ is a $4 \times 4$ skewsymmetric matrix which is the curvature tensor of $S O(5) / S O(4)$. For $H\left(=P_{3}\right)$, we obtain the well-known fact [1]

LEMMA 5.1. A conformal transformation of $S^{4}(1)$ induces a holomorphic bundle automorphism of $P_{3}$.

\section{§6. The complex contact structure of $P_{8}$.}

Let $\left(z^{0}, z^{1}, z^{2}, z^{3}\right)$ be homogeneous coordinates of $P_{3}$. For complex coordinates $\left(z^{1}, z^{2}, z^{3}\right)$ on $\left\{z \in P_{3}: z^{0}=1\right\}$, we define a holomorphic 1-form $\omega$ by

$$
\omega:=d z_{1}-z_{3} d z_{2}+z_{2} d z_{3} \text {. }
$$

Then $\omega$ gives the horizontal distribution $\mathscr{H}$ of $P_{3}$ in the section 5 (see, [4, Proposition 3.1, page 468]). $\omega$ is called the complex contact structure. In this section, we give the differential equation which $\omega$ satisfies.

Let $\mathfrak{n}$ be a unit vertical vector field of the submersion $P_{8} \rightarrow S^{4}(1)$. Then a tensor field $G$ of type $(1,1)$ defined by $G(X)=2 A_{X} \mathfrak{n}$ satisfies

$$
G(\text { vertical vector })=0
$$

$G$ is an endomorphism of $\mathscr{H}_{p}$ which satisfies $G^{2}=-I_{\mathscr{C}_{p}}$. By a simple calculation, we get 


$$
\begin{aligned}
&\left(\tilde{V}_{X} G\right) Y=-\frac{1}{2}\langle X, Y\rangle \mathfrak{n}-\frac{1}{2}\langle J G X, G Y\rangle J_{\mathfrak{n}}+\left\langle\tilde{V}_{X} \mathfrak{n}, J \mathfrak{n}\right\rangle J G(Y), \\
&\left(\tilde{V}_{X} G\right) \mathfrak{n}=\frac{1}{2} X, \\
&\left(\tilde{\nabla}_{V} G\right) X=-\frac{1}{2}\langle V, J \mathfrak{n}\rangle J X-\left\langle\tilde{V}_{X} \mathfrak{n}, J \mathfrak{n}\right\rangle \tilde{\nabla}_{V} \mathfrak{n}+\left\langle\tilde{V}_{V} \mathfrak{n}, J \mathfrak{n}\right\rangle J \tilde{V}_{X} \mathfrak{n},
\end{aligned}
$$

where $X$ and $Y$ are horizontal. $\tilde{V}$ is the connection of $P_{8}$.

\section{§7. Holomorphic isotropic planes of $C^{5}$.}

Let $l$ be a complex plane of $C^{5}$. Then $l$ is called a totally isotropic plane if $\langle X, Y\rangle=0$ for all $X, Y \in l$. It is well known that the space $H_{2}$ of totally isotropic planes is $S O(5) / 1 \times U(2)$ ([2, page 89]). The correspondence is given by

$$
\left(e_{0}, e_{1}, e_{2}, e_{3}, e_{4}\right) \in S O(5) \longrightarrow\left\{B_{1}, B_{2}\right\} \in H_{2},
$$

where $B_{1}=\left(e_{1}+i e_{3}\right) / \sqrt{2}$ and $B_{2}=\left(e_{2}+i e_{4}\right) / \sqrt{2}$. Thus $H_{2}$ is $H\left(=P_{3}\right)$. Furthermore the Hopf fibration is given by

$$
\left\{B_{1}, B_{2}\right\} \longrightarrow B_{1} \wedge B_{2} \wedge \bar{B}_{1} \wedge \bar{B}_{2},
$$

where $B_{1} \wedge B_{2} \wedge \bar{B}_{1} \wedge \bar{B}_{2}$ is a real vector of $\wedge^{4} C^{5}$ (that is, $R^{5}$ ). $H_{2}$ is a Kaehler submanifold in the complex Grassmann manifold and hence is immersed in $P_{9}(2)$ by using Plücker coordinates ([2, (4.5) Proposition, page 89]). $S(5, C)$ acts on $C^{5}$ and hence on $\wedge^{2} C^{5}$ in the standard way. Thus $S O(5, C)$ acts holomorphically on $P_{9}(2)$ and preserves $H_{2}$. Hence $S O(5, C)$ acts holomorphically on $H_{2}$. Let $M$ be a superminimal surface of $S^{4}(1)$, $\chi$ the immersion and $\xi$ the directrix curve of $P_{4}$. Let $\xi$ be a local expression of $\xi$. Then by (2.1), $\xi \wedge \xi^{\prime}$ is an element of $H_{2}$ and gives a global holomorphic map $\tilde{\chi}$ of $M$ into $H_{2}$. By Proposition 2.1, the projection of $\tilde{\chi}$ into $S^{4}(1)$ by $\pi$ is $\chi$. Since the metric of the superminimal surfrce is induced by $\tilde{\chi}, \tilde{\chi}$ is horizontal. Let $A \in S O(5, C)$. Then $A \xi \wedge A \xi^{\prime}$ is totally isotropic and hence the projection of $A \tilde{\chi}$ by $\pi$ gives a superminimal surface. Thus $A \tilde{\chi}$ is horizontal.

\section{§8. Calabi lifting.}

Let $M$ be a Riemannian surface immersed in $S^{4}(1)$ with a complex structure $J^{1}$. We denote by $\langle$,$\rangle and \nabla$ the metric and the connection, respectively. Let $\chi$ be the immersion of $M$ into $S^{4}(1)$. Fix an orientation of $S^{4}(1)$. Then, for a point $x$ of $M, T_{x}\left(S^{4}(1)\right)$ has an orthogonal complex 
structure $J_{x}=J_{x}^{1}+J_{x}^{2}$. By the definition of the twistor space, we obtain a map $\tilde{\chi}$ of $M$ into $P_{3}$ defined by $x \in M \rightarrow J_{x} \in P_{s}$. This map is called the positive Calabi lifting ([15]). The map $\widetilde{\tilde{\chi}}$ defined by the reverse orientation is called the negative Calabi lifting. Let $e_{1}, e_{2}, e_{3}, e_{4}$ be an oriented frame field of $S^{4}(1)$ such that $e_{1}, e_{3}$ is an oriented frame field of $M$. Then $e_{3}=J^{1} e_{1}$ and $e_{4}=J^{2} e_{2}$ hold and hence the positive Calabi lifting is given by:

$$
M \ni x \longrightarrow\left(e_{1}(x), e_{2}(x), e_{3}(x), e_{4}(x)\right) U(2) \in P_{3} .
$$

Let $\gamma$ be a curve of $M$ which satisfies $\gamma(0)=x$. Then we may regard $e_{1}, e_{3}$ as a parallel frame field along $\gamma$ in $M$ and $e_{2}, e_{4}$ as a parallel frame field along $\gamma$ in $N(M)$. Furthermore we denote by $f_{1}, f_{2}, f_{3}, f_{4}$ the parallel frame field along $\gamma$ in $S^{4}(1)$ such that $f_{j}(0)=e_{j}(0)$ for $1 \leqq j \leqq 4$. Therefore there exist functions $a_{j}^{k}$ of the parameter $s$ of $\gamma$ such that

$$
e_{j}=\sum_{j=1}^{4} a_{j}^{k} f_{k}
$$

and hence

$$
\tilde{\chi}(\gamma(s))=\left(f_{1}(s), \cdots, f_{4}(s)\right)\left(a_{j}^{k}(s)\right) U(2) .
$$

Since

$$
\bar{\nabla}_{r_{*(0)}} e_{j}=\sum_{k=1}^{4}\left(a_{j}^{k}\right)^{\prime}(0) e_{k}
$$

holds, we get

$$
\left(\tilde{\chi}_{*} \gamma_{*}(0)\right)^{*}=\tilde{\gamma}_{*}(0) \text { and }\left(\tilde{\chi}_{*} \gamma_{*}(0)\right)^{\nu}=\left(\left(a_{j}^{k}\right)^{\prime}(0)\right)^{\nu} \text {. }
$$

Let $\sigma$ be the second fundamental form of $M$. Then we obtain

$$
\left(\left(a_{j}^{k}\right)^{\prime}(0)\right)^{p}=\left[\begin{array}{ll}
-\left\langle\sigma\left(\gamma_{*}(0), e_{1}\right), e_{2}\right\rangle & -\left\langle\sigma\left(\gamma_{*}(0), e_{1}\right), e_{4}\right\rangle \\
+\left\langle\sigma\left(\gamma_{*}(0), e_{3}\right), e_{4}\right\rangle & -\left\langle\sigma\left(\gamma_{*}(0), e_{3}\right), e_{2}\right\rangle
\end{array}\right],
$$

where $[A: B]$ is an element of $\mathfrak{p}$ such that $A$ is the $(1,2)$-component of $\mathfrak{p}$ and $B$ is the $(1,4)$-component of $\mathfrak{p}$. Now assume that $\gamma(s)$ is a geodesic in $M$ with $\gamma(0)=x$ and $\gamma_{*}(0)=e_{1}$. Let $\gamma(s, t)$ be a family of geodesics with $\gamma(s, 0)=\gamma(s)$ and $\gamma_{*}(s, 0)=e_{3}(s)$. Let $e_{1}(s, t), e_{2}(s, t), e_{3}(s, t), e_{4}(s, t)$ be a frame field along $\gamma(s, t)$, where $e_{1}(s, t), e_{3}(s, t)$ is a parallel frame field along $\gamma(s, t)$ with respect to the connection of $T(M)$ such that $e_{1}(s, 0)=$ $e_{1}(s), e_{3}(s, 0)=e_{3}(s)$ and $e_{2}(s, t), e_{4}(s, t)$ is a parallel frame field along $\gamma(s, t)$ with respect to the connection of $N(M)$ such that $e_{2}(s, 0)=e_{2}(s), e_{4}(s, 0)=$ $e_{4}(s)$. Furthermore let $f_{j}(s, t)$ be a parallel frame field along $\gamma(s, t)$ with 
respect to the connection of $T\left(S^{4}(1)\right)$ such that $f_{j}(s, 0)=f_{j}(s)$. Then there exists a matrix $A(s, t)=\left(A_{j}^{k}(s, t)\right)$ such that

$$
\begin{aligned}
& \left(e_{1}(s, t), e_{2}(s, t), e_{3}(s, t), e_{4}(s, t)\right) \\
& \quad=\left(f_{1}(s, t), f_{2}(s, t), f_{3}(s, t), f_{4}(s, t)\right) A(s, t) .
\end{aligned}
$$

Let $V$ be a vector of $\mathfrak{p}$. Then we have a variation $\tau: I \times I \times I \rightarrow P_{3}$ of $\tilde{\chi}(\gamma(s, t))$ defined by

$$
\tau:(s, t, \theta)=\left(f_{1}(s, t), \cdots, f_{4}(s, t)\right)(\exp \theta V) A(s, t) U(2) .
$$

It is easy to see that, at $x$,

$$
\left(\tilde{\nabla}_{\partial / \partial s} \frac{\partial}{\partial s}\right)^{\nu}=\left(\frac{\partial^{2} A}{\partial s^{2}}\right)^{p}, \quad\left(\tilde{\nabla}_{\partial / \partial t} \frac{\partial}{\partial t}\right)^{\nu}=\left(\frac{\partial^{2} A}{\partial t^{2}}\right)^{\nu}, \quad\left(\tilde{\nabla}_{\partial / \partial s} \frac{\partial}{\partial t}\right)^{\nu}=\left(\frac{\partial^{2} A}{\partial s \partial t}\right)^{p} .
$$

By (8.1), we obtain

$$
\sigma\left(e_{1}(s, 0), e_{1}(s, 0)\right)=\sum_{j=1}^{4} \frac{\partial A_{1}^{j}}{\partial s}(s) f_{j}(s)
$$

and hence

$$
\left(\nabla_{e_{1}}^{\prime} \sigma\right)\left(e_{1}, e_{1}\right)-\sum_{a=1,3}\left\langle\sigma\left(e_{1}, e_{1}\right), \sigma\left(e_{1}, e_{a}\right)\right\rangle e_{a}=\sum_{j=1}^{4} \frac{\partial^{2} A_{1}^{j}}{\partial s^{2}}(0) e_{j}, \quad \text { etc. }
$$

Using the formulas of the submersion ([16]), we obtain

LEMMA 8.1.

$$
\begin{aligned}
& \tilde{\chi}_{*}(X)=\chi_{*}(x)+\frac{1}{2}\left[\begin{array}{ll}
-\left\langle\sigma\left(X, e_{1}\right), e_{2}\right\rangle & -\left\langle\sigma\left(X, e_{1}\right), e_{4}\right\rangle \\
+\left\langle\sigma\left(X, e_{3}\right), e_{4}\right\rangle & -\left\langle\sigma\left(X, e_{3}\right), e_{2}\right\rangle
\end{array}\right] \\
& \tilde{\nabla}_{\partial / \partial} \frac{\partial}{\partial s}=\left\langle\sigma\left(e_{1}, e_{3}\right), e_{4}\right\rangle e_{2}-\left\langle\sigma\left(e_{1}, e_{3}\right), e_{2}\right\rangle e_{4} \\
& +\frac{1}{2}\left[\begin{array}{ll}
-\left\langle\left(\nabla_{e_{1}}^{\prime} \sigma\right)\left(e_{1}, e_{1}\right), e_{2}\right\rangle & -\left\langle\left(\nabla_{e_{1}}^{\prime} \sigma\right)\left(e_{1}, e_{1}\right), e_{4}\right\rangle \\
+\left\langle\left(\nabla_{e_{1}}^{\prime} \sigma\right)\left(e_{1}, e_{3}\right), e_{4}\right\rangle & -\left\langle\left(\nabla_{e_{1}}^{\prime} \sigma\right)\left(e_{1}, e_{3}\right), e_{2}\right\rangle
\end{array}\right] \\
& \tilde{\nabla}_{\partial / \partial t} \frac{\partial}{\partial t}=-\left\langle\sigma\left(e_{1}, e_{8}\right), e_{4}\right\rangle e_{2}+\left\langle\sigma\left(e_{3}, e_{1}\right), e_{2}\right\rangle e_{4} \\
& +\frac{1}{2}\left[\begin{array}{ll}
-\left\langle\left(\nabla_{e_{3}}^{\prime} \sigma\right)\left(e_{3}, e_{1}\right), e_{2}\right\rangle & -\left\langle\left(\nabla_{e_{3}}^{\prime} \sigma\right)\left(e_{3}, e_{1}\right), e_{4}\right\rangle \\
+\left\langle\left(\nabla_{e_{3}}^{\prime} \sigma\right)\left(e_{3}, e_{3}\right), e_{4}\right\rangle & -\left\langle\left(\nabla_{e_{3}}^{\prime} \sigma\right)\left(e_{3}, e_{3}\right), e_{2}\right\rangle
\end{array}\right] \\
& \tilde{V}_{\partial / \partial_{8}} \frac{\partial}{\partial t}=\frac{1}{2}\left(\left\langle\sigma\left(e_{3}, e_{3}\right), e_{4}\right\rangle-\left\langle\sigma\left(e_{1}, e_{1}\right), e_{4}\right\rangle\right) e_{2}
\end{aligned}
$$




$$
\begin{aligned}
& +\frac{1}{2}\left(\left\langle\sigma\left(e_{1}, e_{1}\right), e_{2}\right\rangle-\left\langle\sigma\left(e_{3}, e_{3}\right), e_{2}\right\rangle\right) e_{4} \\
& +\frac{1}{2}\left[\begin{array}{ccc}
-\left\langle\left(\nabla_{e_{1}}^{\prime} \sigma\right)\left(e_{3}, e_{1}\right), e_{2}\right\rangle & -\left\langle\left(\nabla_{e_{1}}^{\prime} \sigma\right)\left(e_{3}, e_{1}\right), e_{4}\right\rangle \\
+\left\langle\left(\nabla_{e_{1}}^{\prime} \sigma\right)\left(e_{3}, e_{3}\right), e_{4}\right\rangle & -\left\langle\left(\nabla_{e_{1}}^{\prime} \sigma\right)\left(e_{3}, e_{3}\right), e_{2}\right\rangle
\end{array}\right] .
\end{aligned}
$$

Let $\omega$ be the Kaehler form of $P_{3}$. Then we obtain

$$
\tilde{\chi}^{*} \omega=\left\langle J \tilde{\chi}_{*} e_{1}, \tilde{\chi}_{*} e_{3}\right\rangle^{*} 1
$$

where ${ }^{*} 1$ is the volume form. Using an $E$-frame in the section 1 , we get

$$
\left\langle J \tilde{\chi}_{*} e_{1}, \tilde{\chi}_{*} e_{3}\right\rangle=K+K_{N}
$$

and hence

$$
\tilde{\chi}^{*} \omega=\left(K+K_{N}\right)^{*} 1
$$

For the negative Calabi lifting, we obtain

$$
\tilde{\tilde{\chi}}^{*} \omega=\left(K-K_{N}\right)^{*} 1 \text {. }
$$

TheOREM 8.1.

$$
\begin{gathered}
\text { degree of } \tilde{\chi}+\text { degree of } \tilde{\tilde{\chi}}=\chi(M), \\
\text { degree of } \tilde{\chi} \text {-degree of } \tilde{\tilde{\chi}}=\chi(N(M)) .
\end{gathered}
$$

Furthermore we obtain

THEOREM 8.2. $\tilde{\chi}$ is totally real if and only if $K+K_{N}=0$. In particular the Calabi lifting of a flat torus isometrically immersed in $S^{4}(1)$ with trivial normal connection is totally real.

Using an $E$-frame, we obtain

$$
\begin{gathered}
\left\langle\tilde{\chi}_{*} e_{1}, \tilde{\chi}_{*} e_{1}\right\rangle=1+(\lambda-\nu)^{2}, \\
\left\langle\tilde{\chi}_{*} e_{1}, \tilde{\chi}_{*} e_{3}\right\rangle=\delta(-\lambda+\nu), \\
\left\langle\tilde{\chi}_{*} e_{3}, \tilde{\chi}_{*} e_{3}\right\rangle=1+(\mu+\nu)^{2}+\delta^{2} .
\end{gathered}
$$

Thus we get

TheOREM 8.3. $\tilde{\chi}$ is conformal at $x \in M$ if and only if $\chi$ is minimal at $x$ or the ellipse of curvature at $x$ is a positive circle.

Furthermore, since the energy function $E$ of $\tilde{\chi}$ is given by

$$
2+(\lambda-\nu)^{2}+(\mu+\nu)^{2}+\delta^{2},
$$


we have the following.

THEOREM 8.4.

$$
E=4 \int\left(1+|\mathfrak{h}|^{2}\right)^{*} 1-4(\chi(M)+\chi(N(M)),
$$

where $\mathfrak{h}$ is the mean curvature vector of $M$.

For a map $f$ of $M$ into $P_{3}$, it is well-known that

the energy of $f \geqq 4 \pi \times \mid$ degree of $f \mid$,

the equality holds if and only if $f$ is holomorphic. This, together with the formula of $E$ for the positive and negative Calabi liftings, implies the following.

COROLlaRY 8.1.

$$
\int\left(1+|\mathfrak{h}|^{2}\right)^{*} 1 \geqq 2 \pi(\chi(M)+|\chi(N(M))|),
$$

the equality holds if and only if the Calabi lifting for the orientation of $N(M)$ with $\chi(N(M)) \geqq 0$ is holomorphic.

This gives a result in [11] for the case that the ambient space is $S^{4}(1)$.

\section{§9. Holomorphic Calabi lifting.}

The Calabi lifting is holomorphic if and only if

$$
J\left(\tilde{\chi}_{*} X\right)=\tilde{\chi}_{*}\left(J^{1} X\right),
$$

which is equivalent to

$$
J\left(\tilde{\chi}_{*} X\right)^{*}=\left(\tilde{\chi}_{*} J^{1} X\right)^{*} \text { and } J\left(\tilde{\chi}_{*} X\right)^{\nu}=\left(\tilde{\chi}_{*} J^{1} X\right)^{\nu} .
$$

Using an $E$-frame and Lemma 8.1, we have $\delta=0$ and $\nu=(1 / 2)(\lambda-\mu)$ and hence we obtain

THeOREM 9.1. (1) The positive Calabi lifting is holomorphic if and only if the ellipse of curvature is a positive circle.

(2) Let $M$ be a holomorphic curve in $P_{3}$ whose image of the Hopf fibration is a regular surface. Then the positive Calabi lifting of the regular surface is a holomorphic map of $M$.

This result is obtained by Atiyah and Lawson (see [11]). 
The horizontal condition of the Calabi lifting is equivalent to $\delta=0$, $\nu=\lambda, \lambda+\mu=0$ in terms of an $E$-frame. Thus, combined with the result of the section 7 , we have

THEOREM 9.2. The poitive Calabi lifting is horizontal if and only if $M$ is a superminimal surface. Furthermore the Calabi lifting is given by the associated map $\Xi_{1}$ of the directrix curve $\Xi$.

Using [17] or the author's unpublished result on the first eigenvalue of Kaehler submanifolds in $P_{n}$, we have

COROLlaRY 9.1. Let $M$ be a not-totally geodesic superminimal surface of $S^{4}(1)$. Then the first eigenvalue of the Laplacian of $M$ is smaller than 2.

COROLlaRY 9.2. $S O(5, C)$ preserves the horizontal distribution $\mathscr{H}$ of $P_{3}$.

Proof. For a point $p \in P_{3}$ and a horizontal holomorphic plane $T$ of $T_{p}\left(P_{3}\right)$, we have the Veronese surface $\chi: S^{2}(1 / 3) \rightarrow S^{4}(1)$ whose Calabi lifting is tangent to $T$ at $p$. Any $A \in S O(5, C)$ makes $A \tilde{\chi}$ horizontal because $A \tilde{\chi}$ is the Calabi lifting of a superminimal surface. Thus $A T$ is a holomorphic plane.

Q.E.D.

\section{\$10. A characterization of horizontal holomorphic curves in $P_{3}$.}

We note that a horizontal holomorphic curve is the positive Calabi lifting of a superminimal surface $M$. Let $e_{1}, e_{3}$ be a frame field of $M$. Then $\tilde{\chi}_{*} e_{1}=\chi_{*} e_{1}$ and $\tilde{\chi}_{*} e_{3}=\chi_{*} e_{3}$. Set $f_{1}=\widetilde{e}_{1}$ and $f_{2}=\tilde{e}_{3}$. For the second fundamental form $\tilde{\sigma}_{2}$ of $\tilde{\chi}$, we have

$$
\sigma_{2}\left(f_{1}, f_{1}\right)=\kappa_{1} \widetilde{e}_{2}, \quad \widetilde{\sigma}_{2}\left(f_{2}, f_{2}\right)=-\kappa_{1} \widetilde{e}_{2}, \quad \tilde{\sigma}_{2}\left(f_{1}, f_{2}\right)=A_{\tilde{e}_{1}} \widetilde{e}_{3}+\kappa_{1} \widetilde{e}_{4} .
$$

Since

$$
A_{\tilde{e}_{1}} \widetilde{e}_{3}=\frac{1}{2}\left(\left\langle R_{\theta_{1} \theta_{3}} e_{k}, e_{j}\right\rangle\right)^{\natural}=0,
$$

we obtain $f_{3}=\widetilde{e}_{2}, f_{4}=\widetilde{e}_{4}$ and $\kappa_{1}=\lambda$ as the Frenet frame field. It follows that

$$
\tilde{\nabla}_{f_{1}} f_{3}=-\kappa_{1} f_{1}+\omega_{3,4}\left(f_{1}\right) f_{4}+\kappa_{2} f_{5} .
$$

Since $A_{f_{1}} f_{3}=(1 / 4)[-1: 0]$, we obtain $\kappa_{2}^{2}=(1 / 4)$. The converse also holds, that is, we have the following. 
THEOREM 10.1. $\quad M$ is a holomorphic curve of $P_{3}$ of constant $\kappa_{2}$ if and only if $M$ is horizontal. In particular $\kappa_{1}=\lambda$, where $\lambda$ is defined for the superminimal surface $\pi(M)$.

Proof. By Lemma 3.1, we obtain $\kappa_{2}=(1 / 2)$. Let $U$ be an open subset of $M$ where a Frenet framd $f_{1}, \cdots, f_{0}$ is defined. Put $\mathfrak{n}=2 f_{\mathfrak{b}}$ and define a tensor field $G$ of $P_{8}$ over $U$ by

$$
G\left(f_{1}\right)=-f_{3}, \quad G\left(f_{2}\right)=f_{4}, \quad G\left(f_{3}\right)=f_{1}, \quad G\left(f_{4}\right)=-f_{2}, \quad G\left(f_{5}\right)=G\left(f_{6}\right)=0 .
$$

Then we obtain

$$
G^{2}=-I \text { over the distribution spanned by } f_{1}, f_{2}, f_{3} \text { and } f_{4}
$$

and

$$
\tilde{\bar{\nabla}}_{f_{j}} \mathfrak{n}=G\left(f_{j}\right)+\omega_{b_{, e}}\left(f_{j}\right) J \mathfrak{n} .
$$

Using $\omega_{8,6}=\omega_{8,4}+\omega_{1,2}$, we find that the differential equation of $(\mathfrak{n}, G)$ is the same one as the complex contact structure of $P_{8}$ satisfies. Making a holomorphic isometry of $P_{3}$ acting on $\omega$, we obtain a new complex contact structure whose initial condition is the same one as $(\mathfrak{n}, G)$. By the uniqueness of solution, the map over $U$ is horizontal in $P_{3}$ with the new complex contact structure and by analiticity of $M, M$ is horizontal.

Q.E.D.

\section{§11. Harmonic Calabi lifting.}

The harmonicity condition of the Calabi lifting is equivalent to

$$
\tilde{\bar{V}}_{\partial / \partial s} \frac{\partial}{\partial s}+\tilde{\bar{\nabla}}_{\partial / \partial t} \frac{\partial}{\partial t}=0 \text {. }
$$

It follows from Lemma 8.1 that the left-hand-side of the above equation is give by

$$
\left[-\left\langle D_{\mathfrak{e}_{1}} \mathfrak{h}, e_{4}\right\rangle:+\left\langle D_{\mathfrak{e}_{1}} \mathfrak{h}, e_{2}\right\rangle\right]
$$

and hence we have

THEOREM 11.1. The positive Calabi lifting is harmonic if and only if $\mathfrak{h}$ is a holomorphic section on $N(M)$.

If $\chi$ is minimal, it is clear that $\chi$ is harmonic, which, together with Theorem 8.2, implies the following.

CoROLLARY 11.1. If $\chi$ is minimal, then the positive and negative 
Calabi lifting are conformal and minimal.

Since $S^{8}(1)$ admits an orientable embedded minimal surface of genus $p$ [14], we obtain

CoROLlaRY 11.2. $P_{8}$ admits an oriented embedded minimal surface of genus $p$ and degree $1-p$.

\section{§12. Isotropic harmonic Calabi lifting.}

Let $M$ be an orientable Riemannian surface with a fixed orientation isometrically immersed in $S^{4}(1)$ with a fixed orientation. Let $\chi$ be the immersion of $M$ into $S^{4}(1)$. Then if $\tilde{\chi}$ is non-holomorphic and isotropic harmonic, the isotropy condition implies that $\tilde{\chi}$ is conformal (see, $[9$, page 237]). By Theorem 8.3, $M$ has the decomposition $M=M_{1} \cup M_{2}$ such that $M$ is minimal on $M_{1}$ and the ellipse of curvature is a positive circle on $M_{2}$. If $M_{2}$ contains an open subset of $M$, then $\tilde{\chi}$ is holomorphic on the open subset and hence so is on $M$ because $\tilde{\chi}$ is analytic. Thus $M_{1}$ is dense in $M$ and hence $M$ is minimal. Fix a point $p$ which has a neighborhood where its ellipse of curvature is not a positive circle. Let $z=x+i y$ be an isothermal coordinate of the neighborhood such that

$$
\frac{\partial}{\partial x}=\frac{\partial}{\partial s} \quad \frac{\partial}{\partial y}=\frac{\partial}{\partial t} \text { at } p,
$$

where $(s, t)$ is the coordinate defined in the section 8 .

LEMMA 12.1. If we set

$$
\frac{\partial}{\partial x}=a \frac{\partial}{\partial s}+b \frac{\partial}{\partial t}, \quad \frac{\partial}{\partial y}=c \frac{\partial}{\partial s}+d \frac{\partial}{\partial t},
$$

then we obtain at $x$

$$
\frac{\partial a}{\partial s}=\frac{\partial d}{\partial s}, \frac{\partial a}{\partial t}=\frac{\partial d}{\partial t}, \quad \frac{\partial c}{\partial s}+\frac{\partial b}{\partial s}=0, \frac{\partial c}{\partial t}+\frac{\partial b}{\partial t}=0,
$$

in particular

$$
\left(\frac{\partial}{\partial s}+i \frac{\partial}{\partial t}\right)(a+i c+i b-d)=0
$$

Set $\phi=\tilde{\tilde{\chi}}$. Now we calculate $D_{\tilde{\chi}}^{\prime} \phi, \quad\left(D^{\prime}\right)^{2} \phi, \cdots, D^{\prime \prime} \phi,\left(D^{\prime \prime}\right)^{2} \phi, \cdots$ to investigate the isotropy condition of $\tilde{\tilde{\chi}}$. 


$$
\begin{aligned}
D^{\prime} \phi= & \frac{1}{2} D_{(\partial / \partial s-i(\theta / \partial t))}^{\prime} \phi=\frac{1}{2}\left(\widetilde{e}_{1}-i \widetilde{e}_{3}\right)^{(1,0)} \\
& +\frac{1}{2}\left[-\left(\sigma\left(E_{1}, \bar{E}_{2}\right), \bar{E}_{2}\right): i\left(\sigma\left(E_{1}, \bar{E}_{1}\right), \bar{E}_{2}\right)\right],
\end{aligned}
$$

where $E_{1}=e_{1}-i e_{3}, E_{2}=e_{2}-i e_{4}$ and $X^{(1,0)}$ is the component of type $(1,0)$ of a vector $X$. By the minimality of $M$, we get

$$
D^{\prime} \phi=\frac{1}{2}\left(\widetilde{e}_{1}-i \widetilde{e}_{3}\right) \text {. }
$$

Similarly we get

$$
D^{\prime \prime} \phi=\frac{1}{4}\left[-\left(\sigma\left(\bar{E}_{1}, \bar{E}_{1}\right), \bar{E}_{2}\right): i\left(\sigma\left(\bar{E}_{1}, \bar{E}_{1}\right), \bar{E}_{2}\right)\right] .
$$

Since

$$
\frac{\partial}{\partial z}=\frac{1}{2}\left((a-i c) \frac{\partial}{\partial s}+(b-i d) \frac{\partial}{\partial t}\right)
$$

holds, we get

$$
\begin{aligned}
\tilde{\bar{\nabla}}_{\partial / \partial z}\left(\phi_{*}\left(\frac{\partial}{\partial z}\right)^{(1,0)}\right) \\
=\frac{1}{8}\left\{\left(\frac{\partial}{\partial s}-i \frac{\partial}{\partial t}\right)(a-i c) \frac{\partial}{\partial s}-\left(\frac{\partial}{\partial s}-i \frac{\partial}{\partial t}\right)(a-i c) i J \frac{\partial}{\partial s}\right. \\
\left.+\left(\frac{\partial}{\partial s}-i \frac{\partial}{\partial t}\right)(b-i d) \frac{\partial}{\partial t}-\left(\frac{\partial}{\partial s}-i \frac{\partial}{\partial t}\right)(b-i d) i J \frac{\partial}{\partial t}\right\} \\
+\frac{1}{4} \tilde{\bar{V}}_{(\partial / \partial s)-i(\theta / \partial t)} \phi\left(\frac{\partial}{\partial s}-i \frac{\partial}{\partial t}\right)^{(1,0)}, \\
\tilde{\bar{\nabla}}_{\partial / \partial \bar{z}} \phi_{*}\left(\frac{\partial}{\partial \bar{z}}\right)^{(1,0)} \\
=\frac{1}{8}\left\{\left(\frac{\partial}{\partial s}+i \frac{\partial}{\partial t}\right)(a+i c) \frac{\partial}{\partial s}-\left(\frac{\partial}{\partial s}+i \frac{\partial}{\partial t}\right)(a+i c) i J \frac{\partial}{\partial s}\right. \\
+\left(\frac{\partial}{\partial s}+i \frac{\partial}{\partial t}\right)(b+i d) \frac{\partial}{\partial t}-\left(\frac{\partial}{\partial s}+i \frac{\partial}{\partial t}\right)(b+i d) i J \frac{\partial}{\partial t} \\
+ \\
+\frac{1}{4} \tilde{\bar{V}}_{(\partial / \partial s)+i(\partial / \partial t)} \phi\left(\frac{\partial}{\partial s}+i \frac{\partial}{\partial t}\right)^{(1,0)} \cdot
\end{aligned}
$$

Using Lemma 8.1 and the minimality, we easily obtain 


$$
\begin{aligned}
D^{\prime 2} \phi= & \frac{1}{8}\left\{\left(\frac{\partial}{\partial s}-i \frac{\partial}{\partial t}\right)(a-i c) \frac{\partial}{\partial s}-\left(\frac{\partial}{\partial s}-i \frac{\partial}{\partial t}\right)(a-i c) i J \frac{\partial}{\partial s}\right. \\
& \left.+\left(\frac{\partial}{\partial s}-i \frac{\partial}{\partial t}\right)(b-i d) \frac{\partial}{\partial t}-\left(\frac{\partial}{\partial s}-i \frac{\partial}{\partial t}\right)(b-i d) i J \frac{\partial}{\partial t}\right\} \\
& +\frac{1}{4}\left(\sigma\left(e_{1}, E_{1}\right), \bar{E}_{2}\right) \widetilde{E}_{2},
\end{aligned}
$$

and

$$
\begin{aligned}
D^{\prime \prime 2} \phi= & \frac{1}{8}\left\{\left(\frac{\partial}{\partial s}+i \frac{\partial}{\partial t}\right)(a+i c) \frac{\partial}{\partial s}-\left(\frac{\partial}{\partial s}+i \frac{\partial}{\partial t}\right)(a+i c) i J \frac{\partial}{\partial s}\right. \\
& \left.+\left(\frac{\partial}{\partial s}+i \frac{\partial}{\partial t}\right)(b+i d) \frac{\partial}{\partial t}-\left(\frac{\partial}{\partial s}+i \frac{\partial}{\partial t}\right)(b+i d) i J \frac{\partial}{\partial t}\right\} \\
& -\frac{1}{4}\left(\sigma\left(e_{1}, \bar{E}_{1}\right), \bar{E}_{2}\right) \widetilde{E}_{2} \\
& -\frac{1}{8}\left[\left(\nabla_{e_{1}}^{\prime} \sigma\right)\left(\bar{E}_{1}, \bar{E}_{1}\right), \bar{E}_{2}\right): i\left(\left(\nabla_{e_{1}}^{\prime}\left(\bar{E}_{1}, \bar{E}_{1}\right), \bar{E}_{2}\right)\right]
\end{aligned}
$$

It follows from Lemma 12.1 that

$$
D^{\prime \prime 2} \phi=-\frac{1}{4}\left(\sigma\left(e_{1}, \bar{E}_{1}\right), \bar{E}_{2}\right) \widetilde{E}_{2}+\text { vertical part }
$$

The isotropy condition implies $\left\langle D^{\prime 2} \phi, D^{\prime \prime 2} \phi\right\rangle=0$ and hence

$$
\left(\sigma\left(e_{1}, E_{1}\right), \bar{E}_{2}\right)\left(\sigma\left(e_{1}, \bar{E}_{1}\right), \bar{E}_{2}\right)=0 \text {. }
$$

For an $E$-frame,

$$
\left(\sigma\left(e_{1}, E_{1}\right), \bar{E}_{2}\right)=\lambda+\nu \text { and }\left(\sigma\left(e_{1}, \bar{E}_{1}\right), \overline{E_{2}}\right)=\lambda-\nu
$$

hold. Since $\lambda \neq \nu$, we get $\lambda+\nu=0$ and hence, for the reverse orientation of $S^{4}(1), M$ is a superminimal surface. Thus we can calculate the vertical of $D^{\prime 2} \phi$ given by

$$
\begin{aligned}
\left(\frac{\partial}{\partial s}\right. & \left.-i \frac{\partial}{\partial t}\right)(a-i c)\left(\frac{\partial A}{\partial s}\right)-\left(\frac{\partial}{\partial s}-i \frac{\partial}{\partial t}\right)(a-i c) i J\left(\frac{\partial A}{\partial s}\right) \\
& +\left(\frac{\partial}{\partial s}-i \frac{\partial}{\partial t}\right)(b-i d)\left(\frac{\partial A}{\partial t}\right)-\left(\frac{\partial}{\partial s}-i \frac{\partial}{\partial t}\right)(b-i d) i J\left(\frac{\partial A}{\partial t}\right) .
\end{aligned}
$$

Since

$$
\left(\frac{\partial A}{\partial s}\right)^{\mathfrak{p}}=[-\lambda: 0] \text { and }\left(\frac{\partial A}{\partial t}\right)^{\mathfrak{p}}=[0: \lambda]
$$


hold, we obtain $J(\partial A / \partial s)^{\natural}=-(\partial A / \partial t)^{p}$. Therefore the vertical part of $D^{\prime 2} \phi$ vanishes and we obtain

$$
D^{\prime} \phi \text { is parallel to } D^{\prime 2} \phi \text {. }
$$

Conversely if $M$ is superminimal for the reverse orientation of $S^{4}(1)$, then (12.1), $\cdots,(12.4)$ hold. Using the induction, we obtain $\left\langle D^{\prime} \phi, D^{\prime \beta} \phi\right\rangle=0$ for $\beta \geqq 1$. Therefore the positive Calabi lifting becomes a non-holomorphic isotropic harmonic map with $r=2$. On the other hand, the negative Calabi lifting is holomorphic. Next we show that the positive Calabi lifting is constructed from the negative Calabi lifting by the method of Eells and Wood. Let $\tilde{\chi}_{U}$ be the local lift of $\tilde{\chi}$, that is, the map of an open set $U$ of $M$ into $C^{4}$ such that the projection of $\tilde{\chi}_{V}$ by $C^{4}-\{0\} \rightarrow P_{8}\left(=C^{4}-\{0\} / C^{*}\right)$ is $\tilde{\chi}$. Let $\widetilde{\widetilde{E}}_{2}$ be the horizontal lift of $\widetilde{E}_{2}$. Then the map $f$ defined by

$$
x \longrightarrow \tilde{\chi}_{U} \longrightarrow\{\tilde{\widetilde{E}}\}
$$

is well defined and gives a holomorphic map by the definition. Using an $E$-frame, we obtain

$$
\begin{aligned}
\widetilde{\bar{V}}_{\partial / \partial_{s}} \widetilde{E}_{2}= & -i \omega_{4,2}\left(e_{1}\right) \widetilde{E}_{2}-\lambda\left(\widetilde{e}_{1}+i \widetilde{e}_{3}\right)+\lambda \widetilde{e}_{1}+i \lambda \widetilde{e}_{3} \\
& +\frac{1}{4}[-1: i] \\
= & -\omega_{4,2}\left(e_{1}\right) \widetilde{E}_{2}+\frac{1}{4}[-1: i] .
\end{aligned}
$$

It is easy to see that, at $x$, the metric induced by $f$ is equal to the metric of $M$, that is, the metric induced by the negative Calabi lifting. Using a Calabi's rigidity theorem [5], the holomorphic curve constructed by the positive Calabi lifting is the negative Calabi lifting. Furthermore it is easy to see that the polar of the negative Calabi lifting is itself.

THEOREM 12.1. Let $\chi$ be a full minimal immersion of an orientable Riemannian surface with a fixed orientation into $S^{4}(1)$ with a fixed orientation. The positive Calabi lifting is non-holomorphic and isotropic harmonic if and only the negative Calabi lifting is holomorphic. Then the $D^{\prime \prime}$-order is 2 and the positive Calabi lifting is constructed by the method of [9] from the negative Calabi lifting. Furthermore the polar of the negative Calabi lifting is itself.

CoROLLARY 12.1. Let $\chi$ be an isometric immersion of $S^{2}$ into $S^{4}(1)$ whose mean curvature vector is a holomorphic section of $N\left(S^{2}\right)$. Then $\chi\left(S^{2}\right)$ is the image of a holomorphic curve in $P_{3}$ by the Hopf fibration. 
Corollary 12.2. Let $\chi$ be an isometric immersion of $T^{2}$ into $S^{4}(1)$ whose mean curvature vector is a holomorphic section of $N(M)$. If $\chi\left(N\left(T^{2}\right)\right)$ is not zero, then $\chi\left(T^{2}\right)$ is the image of a holomorphic curve in $P_{3}$ by the Hopf fibration.

Proof. Using Proposition 7.3 and Corollary 7.7 in [11], we obtain the result.

REMARK. The Veronese surface $S^{2}(1 / 3) \rightarrow S^{4}(1)$ gives the positive Calabi lifting $S^{2}(1 / 3) \rightarrow P_{3}$. Furthermore the negative Calabi lifting is a non-holomorphic isotropic harmonic map: $S^{2}(1 / 6) \rightarrow P_{3}$. Generally we note that the holomorphic immersion $S^{2}(1 / m) \rightarrow P_{m}$ gives non-holomorphic isotropic harmonic maps:

$$
S^{2}\left(\frac{1}{(2 s+1) m-2 s^{2}}\right) \longrightarrow P_{m} \text { for all } 1 \leqq s \leqq m-1 .
$$

In particular, the above is totally real if and only if $m=2 s$.

From Corollary 12.2, we note that a minimal immersion of $T^{2}$ into $S^{4}(1)$ with $\chi\left(N\left(T^{2}\right)\right) \neq 0$ is a superminimal surface. This fact has the following generalization.

THEOREM 12.2. Let $M$ be a compact orientable Riemannian surface of genus $p$ minimally immersed in $S^{4}(1)$ with

$$
|\chi(N(M))| \geqq 2 p-1 \text {. }
$$

Then $M$ is a superminimal surface.

Proof. Assume that $M$ is not a superminimal surface for any orientation. Let $L$ be the complex vector bundle on $M$ defined by assigning to a point $x$ of $M$ the vertical space at $\tilde{\chi}(x)$ of $T\left(P_{8}\right)$. Then using the connection induced from $T\left(P_{8}\right)$, we make $L$ a holomorphic line bundle. Since $\tilde{\chi}(\partial / \partial \bar{z})$ is a non-zero section of $L^{c}$,

$$
\tilde{\chi}\left(\frac{\partial}{\partial \bar{z}}\right) d \bar{z}
$$

defines a global section of $\Omega^{(0,1)} \otimes L . \quad$ It is easy to see that $\tilde{\chi}(\partial / \partial \bar{z})$ is antiholomorphic. Furthermore the curvature form is given by

$$
\frac{1}{2} \tilde{\chi}^{*} \omega
$$

Since $\overline{L \otimes \Omega^{(0,1)}}$ has a global section, the Chern number of $\overline{L \otimes \Omega^{(0,1)}}$ is nonnegative and hence 


$$
\frac{1}{2}\{(\chi(M))+\chi(N(M))\}-2(1-p) \geqq 0 .
$$

Using the negative Calabi lifting, we obtain

$$
|\chi(N(M))| \leqq 2(p-1) .
$$

Q.E.D.

\section{§13. Holomorphic transformations of $P_{3}$.}

Let $M$ be a full, horizontal holomorphic curve in $P_{3}$ and $f$ the map of $M$ into $P_{8}$. Then, in terms of homogeneous coordinates $z^{0}, z^{1}, z^{2}, z^{8}$, $M$ is horizontal if and only if

$$
\left(z^{1}\right)^{\prime}-z^{3}\left(z^{2}\right)^{\prime}+z^{2}\left(z^{3}\right)^{\prime}=0 \text {. }
$$

Let $T=\left(T_{k}^{j}\right)$ be a holomorphic transformation of $P_{3}$. Then $T \cdot f$ is horizontal if and only if

$$
\begin{aligned}
& \left(T_{1}^{1} T_{0}^{0}+T_{1}^{0} T_{0}^{1}+T_{1}^{3} T_{0}^{2}+T_{1}^{2} T_{0}^{3}\right)\left(z^{1}\right)^{\prime} \\
& \quad+\left(T_{1}^{1} T_{2}^{0}-T_{1}^{0} T_{2}^{1}+T_{1}^{3} T_{2}^{2}-T_{1}^{2} T_{2}^{3}\right)\left(\left(z^{1}\right)^{\prime} z^{2}-\left(z^{2}\right)^{\prime} z^{1}\right) \\
& \quad+\left(T_{1}^{1} T_{3}^{0}-T_{1}^{0} T_{3}^{1}+T_{1}^{3} T_{3}^{2}-T_{1}^{2} T_{3}^{3}\right)\left(\left(z^{1}\right)^{\prime} z^{3}-\left(z^{3}\right)^{\prime} z^{1}\right) \\
& \quad+\left(T_{2}^{1} T_{0}^{0}-T_{2}^{0} T_{0}^{1}+T_{2}^{3} T_{0}^{2}-T_{2}^{2} T_{0}^{3}\right)\left(z^{2}\right)^{\prime} \\
& \quad+\left(T_{2}^{1} T_{3}^{0}-T_{2}^{0} T_{3}^{1}+T_{2}^{3} T_{3}^{2}-T_{2}^{2} T_{3}^{3}\right)\left(\left(z^{2}\right)^{\prime} z^{3}-\left(z^{3}\right)^{\prime} z^{2}\right) \\
& \quad+\left(T_{3}^{1} T_{0}^{0}-T_{3}^{0} T_{0}^{1}+T_{3}^{3} T_{0}^{2}-T_{3}^{2} T_{0}^{3}\right)\left(z^{3}\right)^{\prime}=0 .
\end{aligned}
$$

Since

$$
\left(z^{1}\right)^{\prime}, \quad\left(z^{2}\right)^{\prime}, \quad\left(z^{3}\right)^{\prime}, \quad\left(\left(z^{1}\right)^{\prime} z^{2}-\left(z^{2}\right)^{\prime} z^{1}\right), \quad\left(\left(z^{1}\right)^{\prime} z^{3}-\left(z^{3}\right)^{\prime} z^{1}\right)
$$

are the Plücker coordinates of the associated map $f_{1}: M \rightarrow P_{5}$, by horizontality of $f$, we get

$$
f_{1}: M \longrightarrow P_{4} \subset P_{5} \text {. }
$$

$\pi \cdot f$ gives a superminimal immersion of $M$ into $S^{4}(1)$ and its local representation $\xi$ is given by $G_{2} /\left|G_{2}\right|^{2}$. Thus the induced metric of the directrix curve $\Xi$ of $\pi \cdot f$ is given by

$$
\frac{\left|G_{2}\right|^{2}}{\left|G_{1}\right|^{2}} d z d \bar{z}
$$

Using Lemma 2.3, we get the metric $\lambda^{2} d z d \bar{z}$. On the other hand, since $f$ is holomorphic and horizontal, 


$$
D^{\prime} f=\frac{1}{2}\left(\widetilde{e}_{1}-i \widetilde{e}_{3}\right)
$$

$$
D^{\prime 2} f=\text { the part of }\left(\widetilde{e_{1}}-i \widetilde{e_{3}}\right)+\frac{\lambda}{2} \widetilde{E}_{2}
$$

the metric induced by $f_{1}$ is $\lambda^{2} d z d \bar{z}$. Using the result of the section 7 , the directrix curve is the associated curve of the horizontal holomorphic curve. Since the directrix curve is full, we obtain the condition on $T$ :

$$
\begin{aligned}
T_{1}^{1} T_{0}^{0}+T_{1}^{0} T_{0}^{1}+ & T_{1}^{3} T_{0}^{2}+T_{1}^{2} T_{0}^{3}+T_{2}^{1} T_{3}^{0}-T_{2}^{0} T_{3}^{1}+T_{2}^{3} T_{3}^{2}-T_{2}^{2} T_{3}^{3}=0 \\
& T_{2}^{1} T_{0}^{0}+T_{2}^{0} T_{0}^{1}+T_{2}^{3} T_{0}^{2}+T_{2}^{2} T_{0}^{3}=0 \\
& T_{3}^{1} T_{0}^{0}-T_{3}^{0} T_{0}^{1}+T_{3}^{3} T_{0}^{2}-T_{3}^{2} T_{0}^{3}=0 \\
& T_{1}^{1} T_{2}^{0}-T_{1}^{0} T_{2}^{1}+T_{1}^{3} T_{2}^{2}-T_{1}^{2} T_{2}^{3}=0 \\
& T_{1}^{1} T_{3}^{0}-T_{1}^{0} T_{3}^{1}+T_{1}^{3} T_{3}^{2}-T_{1}^{2} T_{3}^{3}=0
\end{aligned}
$$

Thus $T$ transforms any horizontal holomorphic curve into a horizontal holomorphic curve. Let $G$ be the subset of the holomorphic transformations of $P_{3}$ which satisfy $(13.2) \cdots(13.6)$. Then $G$ is closed and hence a Lie subgroup. We see that $\operatorname{dim} G=10$. Since $G$ contains $S O(5, C)$ by Corollary 9.2, $S O(5, C)$ is the identity component of $G$.

THEOREM 13.1. Let $M$ be a full horizontal holomorphic curve in $P_{3}$ and $T$ a holomorphic transformation of $P_{3}$ such that $T \cdot M$ is horizontal. Then $T \in G$.

\section{§14. Critical surfaces in $S^{4}(1)$.}

Let $M$ be an orientable Riemannian surface isometrically immersed in $S^{4}(1)$. Assume that its positive Calabi lifting is holomorphic. Then we find that

$$
\int\left(1+|\mathfrak{h}|^{2}\right)^{*} 1
$$

attains a minimum value among the regularly homotopic immersions of $M$ into $S^{4}(1)$. On the other hand, Weiner posed the following problem: Are the only closed orientable surfaces immersed in $S^{n}$ with centroid 0 which satisfy the Euler-Lagrange equation for (14.1) minimal surfaces in $S^{n}$ ? We gave a counter example [10]. But it is interesting to consider this problem for $M$ with holomorphic, positive Calabi lifting. Now assume 
that $M$ is horizontal in $P_{3}$. Let $T$ be a holomorphic transformation of $P_{3}$ which is close to the identity transformation. Then $T \cdot M$ is a regular surface in $S^{4}(1)$. Using the argument of $\mathrm{Li}$ and Yau [15, Theorem 1, page 273], we obtain a conformal transformation $S$ such that $S \cdot \pi \cdot T(M)$ has the centroid at 0 . It follows from Lemma 6.1 that there exists a holomorphic transformation $Q$ of $P_{8}$ such that $\pi \cdot Q=S \cdot \pi$ and hence $\pi \cdot Q$. $T(M)$ is a regular surface with centroid 0 . If $\pi \cdot Q \cdot T \cdot \tilde{\chi}$ is a minimal immersion, then $\pi \cdot Q \cdot T \cdot \tilde{\chi}$ is a superminimal immersion and hence $Q \cdot T \cdot \tilde{\chi}$ is horizontal. By Theorem 13.1, Q.T $\in G$ holds. Since the dimensions of the holomorphic transformation group of $P_{3}$ and $G$ are 15 and 10 , respectively, the Iwasawa decomposition of the conformal transformation group of $S^{4}(1)$ implies that there exists $T$ such that $Q \cdot T \in G$. Using the examples constructed in [4, Corollary $H$, page 470$]$, we get

TheOREM 14.1. Let $M$ be an orientable Riemannian surface of genus p. Then there exists a non-minimal immersion such that (14.1) attains the minimum among the regularly homotopic immersions of $M$ into $S^{4}(1)$ and the centroid is 0.

\section{§15. Totally real submanifolds in $P_{8}$.}

We gave a characterization of a horizontal and holomorphic curve in $P_{3}$ of constant $\kappa_{2}$ in the section 10. Moreover we found that the basis $\left\{f_{\mathrm{b}}, f_{\mathrm{B}}\right\}$ of the second normal bundle is one of the tangent space of the fiber of $P_{8}$. Let $T_{r}$ be the tube of radius $r$ in the direction of the second normal bundle. In this section, we investigate $T_{r}$.

Let $(\phi, \xi, \eta,\langle\rangle$,$) be the Sasakian structure of S^{7}(1)$ (See, for example, [18].) and $\bar{\pi}$ be the Hopf fibration of $S^{7}(1)$ onto $P_{8}(4)$. Then for a horizontal holomorphic curve, the second curvature is 1 . Let $\bar{M}$ be the induced bundle over $M$ and $\bar{N}_{2}$ the horizontal lift of the second normal bundle of $M$. We denote by $\bar{T}_{r}$ the tube of $\bar{M}$ in the direction of $\bar{N}_{2}$. Then $\bar{\pi}\left(\bar{T}_{r}\right)$ is $T_{r}$. Let $U$ be an open set where $\left\{f_{s}, f_{\theta}\right\}$ is well-defined. Thus $\bar{T}_{r}$ is locally given by

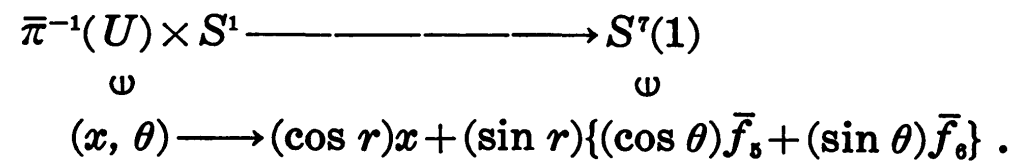

Therefore we obtain

$$
\begin{aligned}
F_{r *} \bar{f}= & (\cos r) \bar{f}_{1}-(\sin r)(\cos \theta) \bar{f}_{8}+(\sin r)(\cos \theta) \omega_{b, 6}\left(f_{1}\right) \bar{f}_{\mathrm{b}} \\
& -(\sin r)(\sin \theta) \bar{f}_{4}-(\sin r)(\sin \theta) \omega_{b, \theta}\left(f_{1}\right) \bar{f}_{\mathrm{b}},
\end{aligned}
$$


etc, which imply that $T_{r}$ is totally real if and only if

$$
\left\{\bar{\pi}_{*} F_{r *} \bar{f}_{j}, \bar{\pi}_{*} F_{*} \frac{\partial}{\partial \theta}: j=1,2\right\}
$$

is perpendicular to

$$
\left\{J \bar{\pi}_{*} F_{r^{*}} \bar{f}_{j}, J \bar{\pi}_{*} F_{r^{*}} \frac{\partial}{\partial \theta}: j=1,2\right\} .
$$

Using the properties of the Sasakian structure, we can investigate the totally real codition. Furthermore, by a long calculation, we obtain

THEOREM 15.1. $\quad T_{r}$ is totally real and minimal in $P_{3}$ if and only if $r=\pi / 2$.

We can calculate the Chern number of the second normal bundle. Since

$$
d \omega_{5,8}=3 d \omega_{12}+\left(\Delta \log \kappa_{1}\right) \omega_{1} \wedge \omega_{2}
$$

holds, it follows from the result in the section 3 that the curvature of the normal bundle is 1 . Hence we obtain

$$
c_{1}=\frac{1}{2 \pi} \text { Volume }(M) \text {. }
$$

From the example $\xi_{k 2}$ of Barbosa [2, page 101], we obtain

COROLLARY 15.1. The circle bundle of even Chern number $\geqq 6$ can be immersed in $P_{3}$ as a totally real and minimal submanifold.

\section{References}

[1] M. F. Atiyah, N. J. Hitchin and I. M. Singer, Self-duality in four-dimensional Riemannian geometry, Proc. Roy. Soc. London Ser. A, 362 (1978), 425-461.

[2] J. L. M. Barbosa, On minimal immersions of $S^{2}$ into $S^{2 m}$, Trans. Amer. Math. Soc., 210 (1975), 75-106.

[3] D. E. BLAIRE and K. OGIUE, Geometry of integral submanifolds of contact distribution, Illinois J. Math., 19 (1975), 269-276.

[4] R. L. BRYANT, Conformal and minimal immersions of compact surfaces into the 4-sphere, J. Differential Geom., 17 (1982), 455-473.

[5] E. CALABI, Isometric imbedding of complex manifolds, Ann. of Math., 58 (1953), 1-23.

[6] B. Y. Chen and K. OGIUE, On totally real submanifolds, Trans. Amer. Math. Soc., 193 (1974), 257-266.

[7] S.S. Chern, On the minimal immersions of the two sphere in a space of constant curvature, Problems in Analysis, Princeton Univ. Press, Princeton, (1970), 27-40.

[8] S.S. Chern and E. A. Spanier, A theorem on orientable surfaces in four-dimensional space, Comment. Math. Helv., 25 (1951), 205-209. 
[9] J. EeLLs and J.C. Wood, Harmonic maps from surfaces to complex projective spaces, Adv. in Math., 49 (1983), 217-263.

[10] N. EJIRI, A counter example for Weiner's open question, Indiana Univ. Math. J., 31 (1982), 209-211.

[11] I. V. GuAdalupe and L. Rodriguez, Normal curvature of surfaces in space forms, Pacific J. Math., 106 (1983), 95-102.

[12] R. HARveY and H. B. Lawson, JR., Calibrated geometries, Acta Math., 148 (1982), 47-157.

[13] H. B. LAwson, JR., The Riemannian Geometry of holomorphic curves, the Proceeding of Carolina conference on holomorphic mappings and minimal surfaces (Chapel Hill, N. C., 1970), Department of Math. Univ. of North Carolina, Chapel Hill, N.C., 1970, 86-106.

[14] H. B. Lawson, Complete minimal surfaces in $S^{3}$, Ann. of Math., 92 (1970), 335-374.

[15] P. LI and S. T. YAU, A new conformal invariant and its applications to the Willmore conjecture and the first eigenvalue of compact surfaces, Invent. Math., 69 (1982), 269-291.

[16] B. O'NeiLl, The fundamental equations of a submersion, Michigan Math. J., 13 (1966), 459-469.

[17] A. Ros, Spectral geometry of $C R$-minimal submanifolds in the complex projective space, Kodai Math. J., 6 (1983), 88-99.

[18] S. TANNo, Sasakian manifolds with constant $\phi$-holomorphic sectional curvature, Tôhoku Math. J., 21 (1969), 501-507.

[19] J. L. VeRDIER, 2-dimensional $\sigma$-models, preprint.

[20] Y.C. WoNG, Contributions to the theory of surfaces in a 4-space of constant curvature, Trans. Amer. Math. Soc., 59 (1946), 467-507.

Present Address:

Department of Mathematics

Faculty of Sciences

TOKYo Metropolitan University

Fukazawa, Setagaya-kU, Tokyo 158 